\section{The Biology of the Coccidia}

\section{Edited by Peter L. Long}

The Biology of the Coccidia is the updated version of The Coccidia edited by the late $\mathrm{Dr}$ Hammond and Dr Long. Since the publication of the first edition there has been much new research on this group of parasites including the discovery that some species are parasites of man as well as other animals. This book has been thoroughly revised and includes a new chapter on chemotherapy and new details of life cycles of several species of Sarcocystis and

Toxoplasma. There are several new contributors. The contributors are all leading authorities in the field.

$£ 45$ boards 512 pages

Publication May

\section{Plant Growth Curves}

A functional approach to plant growth analysis

\section{Roderick Hunt}

A review of the theory, practice and applications of the use of fitted mathematical functions in plant growth analysis. The approach taken is very broad and includes an extended coverage of the philosophical and scientific links between this topic and related fields of activity in plant science. £8. 75 paper 240 pages

Publication June

\section{Optima for Animals}

\section{R. McNeill Alexander}

Optimization theory is the branch of mathematics concerned with finding those structures and behaviours that are in some sense the best possible, and is therefore a very appropriate tool for trying to discover why animals have evolved in particular ways.

$£ 5.25$ paper 120 pages

Publication June

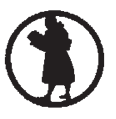

Edward Arnold

41 Bedford Square London WC1B 3DQ death, Einstein wrote a tribute to him. But in spite of this Machian input, a clear-cut manifestation of Mach's principle failed to emerge in any very obvious way from relativity theory. Over the decades, many theoretical physicists have struggled to find formulations of Mach's principle that would mesh easily with either the theory of relativity, or some close variant. It is a task as yet unfinished, but it remains as compelling as ever. At issue is one of the fundamental properties of space-time structure, and the nature of motion.

This timely and most welcome book contains a discussion of the evolution of the space-time concept from Aristotle to Einstein (and beyond), and presents a detailed and up-to-date analysis of Mach's principle in a modern setting. The authors conclude that "At best, Mach's principle has been expressed in general relativity as a selection rule. To go beyond this ... one may have to give up general relativity",

However, this book is far from a catalogue of failures. It is a comprehensive and accurate survey of the physics of space and time and of the philosophical overtones which accompany it. The theory of relativity, both the special and general versions, obviously plays a central role in these ideas, and the reader will find discussions of black holes and details of gravitation experiments as well as a careful introduction to the more elementary aspects of space-time structure.

Mach's principle is an intriguing context in which to learn relativity and space-time physics, as we!l as an end in itself. I can thoroughly recommend this book to undergraduates learning general relativity, as well as to researchers who share the authors' fascination with one of science's age-old mysteries.

Paul Davies is Professor of Theoretical Physics at the University of Newcastle upon Tyne. His most recent book (with N.D. Birrell) is Quantum Fields in Curved Space (Cambridge University Press, 1982).

\title{
The mixed blessing: oxygen in biology
}

\section{Barry Halliwell}

Oxygen and Living Processes: $A n$ Interdisciplinary Approach. Edited by Daniel L. Gilbert. Pp.401. ISBN 3-540-90554-5/0-387-90554-5. (SpringerVerlag:1981.) DM135, \$61.40.

THIS colourless, odourless gas is an extreme fire hazard and is very toxic to some organisms at minute concentrations. Its toxicity to human beings is much slower-acting, but nevertheless over a period of many years it produces a progressive oxidative deterioration that results inevitably in death. Only the swimbladders of certain fishes can tolerate it at high concentrations, although turtles and crocodiles are fairly resistant to its toxic effects unless they are placed in warm water.

The above description, using information taken from this book, applies of course, to oxygen. Oxygen is essential for efficient energy production by oxidation of foodstuffs in aerobic animals, but only its peculiar chemistry protects us from instant "spontaneous" combustion. The same chemistry dictates that the oxygen reduction accompanying substrate oxidation must proceed via partially reduced oxygen intermediates on the pathway to water. Such intermediates as superoxide and hydroxyl radical are particularly noxious and so aerobic cells are equipped with many defences against them, including superoxide dismutase, catalase, glutathione and/or ascorbate peroxidases and vitamin $E$. The cytochrome oxidase complex of mitochondria is carefully designed not to allow the escape of such intermediates from its active site.
As its title declares, this book is indeed an interdisciplinary approach to oxygen. All of the items I have mentioned are covered in detail, and in addition there are chapters on photosynthetic oxygen production, the geological history of the oxygen in the Earth's atmosphere, oxygencarrying pigments, kinetics of oxygen diffusion, the history of the discovery of oxygen, and the medical uses (and abuses) of the gas, covering blindness in babies, pulmonary oxygen toxicity and hyperoxygenation in the treatment of gas gangrene and cancer of the head and neck. The contributions are well-written, all of them by experts in specific fields, and the book has been produced in an attractive style with the minimum of errors.

I do have some minor niggles, however. Much of Chapter 1, a history of the discovery of oxygen, is merely an overcondensed - and rather boring - list of names and dates; for example, it is impossible to do justice to the complex physiology expounded by Galen in a few lines. In Chapter 6, on photosynthesis, I doubt whether non-biologists will really understand what exactly are photosystem I, photosystem II and Q. In addition, the order of the chapters is sometimes a bit illogical.

These comments are not meant to detract from what is an excellent book. It is truly "everything you wanted to know about oxygen, but didn't know whom to ask'.

Barry Halliwell is a Lecturer in Biochemistry at King's College, University of London, and author of Chloroplast Metabolism (Clarendon) Oxford University Press, 1981). 